\title{
Trivium
}

Revue franco-allemande de sciences humaines et sociales - Deutsch-französische Zeitschrift für Geistesund Sozialwissenschaften

11 | 2012

Gustave Flaubert. A l'Orient du réalisme

\section{Flauberts Realismus}

\section{Einleitung}

Barbara Vinken

\section{OpenEdition}

\section{Journals}

Édition électronique

URL : http://journals.openedition.org/trivium/4260

DOI : $10.4000 /$ trivium.4260

ISSN : 1963-1820

\section{Éditeur}

Les éditions de la Maison des sciences de l'Homme

\section{Référence électronique}

Barbara Vinken, «Flauberts Realismus », Trivium [Online], 11 | 2012, online erschienen am 29 Juni 2012, abgerufen am 22 September 2020. URL : http://journals.openedition.org/trivium/4260 ; DOI : https://doi.org/10.4000/trivium.4260

Ce document a été généré automatiquement le 22 septembre 2020

\section{(c) $(1) \&$}

Les contenus des la revue Trivium sont mis à disposition selon les termes de la Licence Creative Commons Attribution - Pas d'Utilisation Commerciale - Pas de Modification 4.0 International. 


\title{
Flauberts Realismus
}

\author{
Einleitung \\ Barbara Vinken
}

1 Lassen Sie mich mit einem Zitat Flauberts beginnen, das vielleicht selbst ausgesprochene Flaubertkenner überrascht. 1852 schreibt Flaubert an Louise Colet: „Voilà ce que tous les socialistes du monde n'ont pas voulu voir, avec leur éternelle prédication matérialiste. Ils ont nié la Douleur, ils ont blasphémé les trois quarts de la poésie moderne, le sang du Christ qui se remue en nous. - Rien ne l'extirpera, rien ne le tarira. Il ne s'agit pas de le dessécher, mais de lui faire des ruisseaux. Si le sentiment de l'insuffisance humaine, du néant de la vie venait à périr (ce qui serait la conséquence de leur hypothèse), nous serions plus bêtes que les oiseaux, qui au moins perchent sur les arbres. $\aleph^{1}$ Das ist erstaunlich: Die moderne Dichtung - zumindest jene, die dieses Namens würdig sein soll - wäre demnach ein antisozialistisches Manifest. Warum? Weil sie nichts anderes als ein Gefäß für das Blut Christi ist. Die moderne Dichtung ist - im Namen des vom Gekreuzigten vergossenen Blutes, der Nichtigkeit des Lebens und der Unzulänglichkeit alles Menschlichen - ein Gegendiskurs zu den leeren und, schlimmer, blasphemischen Heilsversprechen der Sozialisten jeder Couleur. Flaubert interpretiert die Praxis der modernen Dichtung als eine Form der imitatio Christi, die wie jede Nachahmung ein stark körperliches Moment hat: Der eigene Körper nimmt den Schmerz eines anderen Körpers auf sich. In unseren Adern fließt nicht unser eigenes Blut, sondern das Blut Christi, das für uns geopfert wurde: »le sang du Christ qui se remue en nous«. Nur das Blut, das leidend hingegeben wurde, ist ewig. Außerhalb unserer Körper existiert ein anderes Medium für das Fließen des christlichen Blutes, ein anderes Gefäß: die moderne Dichtung. ${ }^{2}$

2 Aber was machen die Vögel hier, die klüger als die, die Heilsversprechungen aufsitzen, "perchent sur les arbres«? Sie folgen Christus nach, "perché sur l'arbre de la croix« (Apg 10, 39). In einem sehr hegelianischen Moment interpretiert Flaubert die Welt und die Schöpfung als Erinnerung und Inszenierung der Kreuzigung. Es ist die implizite, im Wortwitz versteckte Anspielung, "perché sur les arbres«, "perché sur l'arbre de la croix«, die zeigt, wie vertraut Flaubert dieser Gedanke war. 
Hegel bestimmt die Kreuzigung Christi als eine Urszene dessen, was er romantische Kunst nannte. Im Gegensatz zur klassischen Kunst ist die romantische Kunst Kunst unter der Bedingung des Christentums. Die Urszene romantischer Kunst ist die Fleischwerdung. Für Hegel ist die Fleischwerdung ein Akt des souveränen Selbstverzichts, der Entäußerung - Kenosis - des Gottes, der in Christus Mensch geworden ist. Diese radikale Selbsterniedrigung findet im schändlichen Tod am Kreuz ihren höchsten Ausdruck. Nach Hegel liegt die Besonderheit der klassischen Kunst in ihrer Idealität, jener idealen Schönheit, "welche die äußere Anschauung über die Zeitlichkeit und die Spuren der Vergänglichkeit weghebt, um die blühende Schönheit der Existenz an die Stelle ihrer sonstigen verkümmerten Erscheinung zu setzen «. ${ }^{3}$ In der klassischen Kunst geht der Geist ganz ins Körperliche ein. Die Schönheit der Seele spiegelt sich in der Schönheit des Körpers. „Die griechische Schönheit zeigt das Innere der geistigen Individualität ganz in deren leiblicher Gestalt, Handlungen und Begebnisse hineingebildet, im Äußeren ganz ausgedrückt und selig darin lebend. ${ }^{4}$ Die Urszene romantischer Kunst hingegen liegt in der Entfremdung Gottes in der Gestalt des Menschlichen. Die Form kann also nicht mehr wie in der klassischen Kunst den Grund, die Substanz darstellen. "Die Versöhnung mit dem Absoluten ist im Romantischen ein Akt des Inneren, welcher zwar im Äußeren erscheint, aber das Äußere selbst in seiner realen Gestalt nicht zum wesentlichen Inhalt und Zweck hat. ${ }^{5}$

In der romantischen Kunst erscheint dieser »Akt des Inneren« in der objektiven Wahrheit sinnlicher Darstellungen paradox: Er zeigt sich in der Form des Unvollendeten, des Hässlichen oder, radikal ausgedrückt, in der Nichtigkeit und Vernichtung des Scheins als Schein. ${ }^{6}$ Für die romantische Kunst liegt der Triumph der Darstellung in der Ohnmacht der Darstellung, in der Darstellung dessen, was sich der Darstellbarkeit entzieht: "Das Innere in diesem Verhältnis, so auf die Spitze hinausgetrieben, ist die äußerlichkeitslose Äußerung, unsichtbar gleichsam nur sich selber vernehmend, ein Tönen als solches ohne Gegenständlichkeit und Gestalt, ein Schweben über den Wassern, ein Klingen über einer Welt, welche in ihren und an ihren heterogenen Erscheinungen nur einen Gegenschein dieses Insichseins der Seele aufnehmen und widerspiegeln kann. « ${ }^{7}$

5 Auch wenn Auerbach dies nicht ausdrücklich sagt, schreiben sich seine Versuche zum Realismus - der nach Hegel der romantischen Kunst zuzurechnen ist - in die Tradition der hegelianischen Ästhetik ein. Genauso wie Hegels hat Auerbachs Ansatz den unschätzbaren Vorteil, von vornherein die Frage nach der Darstellung von Wirklichkeit auszuklammern. Auerbach interessiert sich vielmehr für die literarischen Verfahren, die Wirklichkeit produzieren, um mit Blumenberg $\mathrm{zu}$ sprechen, für die Möglichkeitsbedingungen der Wirklichkeit ${ }^{8}$, oder, um es mit Barthes zu sagen, für den "effet de réel «. ${ }^{9}$ Wie Hegel verhandelt Auerbach den Realismus grundsätzlich als Ausfaltung einer paradoxen Figur: der Fleischwerdung des Wortes, der Menschwerdung Gottes. ${ }^{10} \mathrm{In}$ diesem rein formalen Sinne ist die romantische Kunst eine christliche Kunst. Das heißt, dass die in dieser Tradition verfasste Dichtung eine verborgene Matrix einer Wirklichkeit hat, deren Konzeptualisierung in einem bestimmten historischen Moment erfolgte, nämlich in den Texten der Evangelien. Flaubert kennt dieses evangelische Erbe nur allzu gut, ja, er wird davon umgetrieben.

6 Folgt man Auerbachs unter anderem an Flauberts Madame Bovary ${ }^{11}$ entwickelter Theorie des Realismus, so besagt diese, dass der Realismus die klassische, bis ins 18. Jahrhundert hinein gültige Gattungspoesie, welche hohen Rang mit hohem Stil koppelt, 
kippt. Die niedrigen Leute und die niedrigen Dinge des täglichen Lebens werden nicht verlacht. Sie sind nicht Gegenstand der Komödie, sondern werden ernst genommen, ernsthaft dargestellt, ja, sie können tragisch sein. Die Schilderung ihres Lebens erweckt Gefühle, die sonst nur dem erhabenen Stil zugeordnet werden.

7 Diese Stilentwicklung beginnt nicht erst im 19. Jahrhundert, sondern bereits, wie Auerbach in seinen Untersuchungen zur lateinischen Spätantike schreibt, mit dem Christentum und dessen Umstoßen der antiken Rhetorikhierarchie. Der Einbruch des Christentums in die heidnische Welt wurde als radikaler Bruch mit der gesamten rhetorischen und literarischen Tradition wahrgenommen. In der Nachfolge Hegels ist dieser Bruch ästhetisch gedeutet worden. So spricht Alexandre Leupin in Anlehnung an Kojève, von einer "coupure épistémologique chrétienne«, die Jean-Claude Milner als Kojèvesches Theorem folgendemaßen nachformuliert: "Il n'y a jamais aucune synonymie entre une notion appartenant au système de pensée païen et une notion chrétienne, c'est à dire moderne.« Und Leupin erklärt: »L'effort d'un Tertullien, par exemple, n'est autre qu'une vaste tentative d'homonymiser les noms de l'ancienne rhétorique pour leur faire dire autre chose - la réalité/vérité de l'Incarnation. ¿12 $^{12} \mathrm{Dieser}$ Bruch wird durch ein Paradox im Kern der christlichen offenbarung motiviert: Die Evangelien nämlich erzählen die unerhörte Geschichte eines (als Messias angekündigten) Gottes, der Mensch wird. Boethius, der um 512 gegen zwei Häresien Contra Eutychen et Nestorium schreibt, erklärt das logische Paradox der Inkarnation so: "Quelle grande et nouvelle chose - unique et non-répétable en aucune époque - que la nature de celui qui est seul Dieu se joigne à la nature humaine (entièrement différente, elle, de Dieu), et forme ainsi une seule personne par la conjonction (copulatione) de natures différentes! «13 Aber damit nicht genug. Dieser Gott wird zu allem Überfluss nicht in einem Palast, sondern in einem Stall, unter den Ärmsten der Armen, geboren. Armut ist die entscheidende Signatur dieser Ankunft, dieser Fleischwerdung. Von Anfang an kennzeichnete das Christentum ein Beharren auf dem Niedrigen und dem Verachteten in einer äußerst stratifizierten und hierarchisierten Gesellschaft. In diesem Sinn ist der Realismus der Armen eine ursprünglich christliche Erfindung.

Doch die strukturell entscheidende Frage liegt anderswo. Der Tod am Kreuz ist die Matrix für die Darstellung des in dieser Welt Fleisch gewordenen Gottes. ${ }^{14} \mathrm{Er}$, der Schöpfer aller Dinge, beschließt seine Existenz verurteilt, geschlagen, verhöhnt, verlacht, bespuckt durch den verworfensten aller Tode..$^{15}$ Der Triumph der Darstellung dieses Kreuzes ist ein Triumph, in dem die antike Ökonomie der Stillagen, die Unterscheidung zwischen dem Niedrigen und dem Erhabenen, zusammenbricht. Sie offenbart ein Moment des Undarstellbaren, welches auf einer unerhörten Ebene theologischer Abstraktion das zusammenfasst, was in der einfachen Prophezeiung eines Messias ausgedrückt worden war.

Die Ausfaltung der romantischen Kunst in Hegels System ist nichts anderes als die Erklärung dieser Matrix. In seiner post-theologischen Lektüre von Luthers reformatorischem Ansatz erscheint die Schönheit des Dargestellten auf sublime Weise, weil sie sich in der Nichtigkeit der Welt verbirgt: "Christus humilis, vos superbi«. Auerbach bringt in diesem Zusammenhang drei Momente zusammen: 1. die Niedrigkeit des Stils der Bibel - sermo humilis -, die nicht von gelehrten Rhetoren, sondern von - so musste es dem gebildeten Publikum erscheinen - fanatischen, halbgebildeten Sektierern in einem seltsam barbarischen, geschmacklosen Stil geschrieben wurde und von kindisch absurdem Aberglauben nur so strotzte; 2. die Niedrigkeit des 
Gegenstandes, die dem Stil entspricht und 3. die Ungebildetheit derer, an deren Ohr oder eher Herz die frohe Botschaft dringen soll.

Auerbach zitiert als Beispiel für die humilitas der Inkarnation den Paulusbrief an die Philipper, in dem Paulus von der "formam servi« spricht, die Jesus annahm, um den Tod eines Sklaven, den Tod am Kreuz zu sterben (Phil. II, 5-9). ${ }^{16}$ Diese Erniedrigung ins Menschliche - Kenosis - hat Luther mit »Nichtigkeit« wiedergegeben; Hegel übersetzt den griechischen Begriff der Kenosis mit »Entäußerung«. Auerbachs Realismus, der den christlichen sermo humilis beerbt, ist in Hegels Begrifflichkeit ein kenotischer Stil extremer Entäußerung.

Was verbindet nun eine kenotische Poetik, die nach Hegel die romantische oder moderne Kunst charakterisiert, mit Flauberts Realismus - mit dem >kryptischen Realismus (P.-M. de Biasi) der Éducation sentimentale und der `Kitschwelt` (M. Föcking) Emma Bovarys? Was bedeutet die Definition der modernen Dichtung, die Flaubert in seinem Brief an Louise Colet gibt? Bereits G. Wildgruber hat Flauberts Schreibpraxis, seine Arbeit am Stilideal der impersonnalité, in der Tradition einer spezifischen imitatio Christi beschrieben, die in der französischen Spiritualität des 17. Jahrhunderts ihre letzten großen Vorbilder hatte. Flaubert war, das ist bekannt, ausgesprochen antiklerikal. A. Bouviers Beitrag "Jéhovah égale Moloch" gibt hier nur ein Beispiel für den literarischen furor, mit dem Flaubertin der Darstellung der idolatrischen Kulte Karthagos nicht nur die jüdischen Kulte, sondern zuletzt römisch-katholische Praktiken der Marien-, Heiligen- und Papstverehrung anprangert. Und obwohl Antiklerikalismus, auch das ist hinlänglich bekannt, Religiosität nicht unbedingt ausschließt, soll im Folgenden nicht nahegelegt werden, Flaubert habe sich am Rande einer Konversion befunden, wie Thibaudet vorgeschlagen hat.

Aber in dem sonderbaren "religious turn« der laizistischen République Française, die ihre Apostel in den Literaturprofessoren und ihre Kirchenväter in den kanonischen Autoren der Grande Nation hat, ist in Flauberts Schreiben etwas verdrängt worden. Seine écriture steht - der Hoffnung republikanischer Professoren zum Trotz - der christlichen Matrix, die Hegel in der Kunst der Moderne festgemacht hat, weder neutral gegenüber, noch ist sie in Opposition zu oder gar jenseits dieser Matrix anzusiedeln. Flaubert entwickelt eine Poetik der Kenosis, der Entäußerung und Erniedrigung, um das Skandalon des Evangeliums in neuer, verschärfter Ambivalenz zu inszenieren. ${ }^{17}$ Flauberts Realismus ist die Praxis einer poetischen Kenosis, deren wirklichkeitsbildendem Prinzip die hier versammelten Beiträge nachspüren.

Der Flaubert, wie er in vielen der hier versammelten Beiträge aufscheint, ist ein anderer Flaubert. Dieser andere Flaubert verdankt sich einer von der ANR und DFG geförderten Zusammenarbeit des CNRS und der LMU zu »Flaubert, die Antike, die Religionen «. Diese Forschergruppe hat die bisher nur angedeuteten Bezüge zwischen der europäischen Mythenforschung und Flauberts Werk offengelegt und so eine bisher kaum erkannte Tiefendimension seines Werkes sichtbar gemacht, die sicher nicht auf den Nenner der Säkularisation zu bringen ist.

\section{Textauswahl}

Bouvier, Agnès: "Jéhovah égale Moloch: une lecture santireligieuse de Salammbô«, Romantisme, 136, 2007, S. 109-120. 
Dufour, Philippe: «Salammbô, un tigre de marbre«, in: Gustave Flaubert 5, hg. v. G. Séginger, Paris / Caen, 2005, S. 193-214.

De Biasi, Pierre-Marc: ")Qu'est-ce que cela veut dire, la réalité?^ Le cryptage du réel dans L'Éducation sentimentale«, in: Vinken, B. / Fröhlicher, P. (Hg.): Le Flaubert réel, Tübingen: Niemeyer, 2009, S. 61-78.

Föcking, Marc: »Arkadien - eine Kitschwelt? Schwundstufen des Arkadischen in Flauberts Madame Bovary«, in: Friedlein, R. / Poppenberg, G. / Volmer, A. (Hg.): Arkadien in den romanischen Literaturen, Heidelberg: Winter, 2008, S. 483-492.

Koppenfels, Martin von: »Flauberts Hand. Strategien der Selbstimmunisierung «, Poetica, 34, 2002, S. 171-191.

9 Matthey, Cécile: »Itinéraire d'une croyance: Hérodias«, in: Neefs, J. (Hg.): Savoirs en récit, Bd. II: Éclats de saviors: Balzac, Nerval, Flaubert, Verne, les Goncourt, Presses Universitaires de Vincennes, 2010, S. 85-92.

Triare, Sylvie: »Le défilé des dieux, de l'idole primitive à Loulou«, in: Herschberg Pierrot, A. (Hg.): Flaubert: les pouvoirs du mythe, avant-propos de B. Vinken et P.-M. de Biasi, Publication Fractal, ANR-DFG. [Actes du séminaire Flaubert 2008-2009, ITEM-Paris 8-ENS], Paris: Éditions Archives Contemporaines, im Druck.

21 Vinken, Barbara: "Sermo humilis: Realismus und Christentum» und »Notre-Dame de Rouen: Flauberts Kathedrale«, aus: Flaubert. Durchkreuzte Moderne, Frankfurt/M.: Fischer, 2009, S. 350-374.

Wildgruber, Gerald: »Kunst - Religion - Wissenschaft. Zur Konstellation dreier Terme im Spätwerk Flauberts«, Hofmannsthal Jahrbuch,7, 1999, S. 307-344.

\section{BIBLIOGRAPHIE}

Auerbach, Erich (1946) : Mimesis. Dargestellte Wirklichkeit in der abendländischen Literatur, Bern: Francke.

Auerbach, Erich (1958) : Literatursprache und Publikum in der lateinischen Spätantike und im Mittelalter, Bern: Francke.

Blumenberg, Hans (1964): »Wirklichkeitsbegriff und Möglichkeit des Romans«, in: Jauss, H. R. (Hg.): Nachahmung und Illusion, München: Fink (Poetik und Hermeneutik 1).

Culler, Jonathan (1975): Structuralist Poetics, London / Ithaca: Routledge and Kegan Paul / Cornell University Press.

Flaubert, Gustave (1980): Correspondance, Bd. II, hg. v. J. Bruneau, Paris: Gallimard.

Hegel, Georg Wilhelm Friedrich (1971): Ästhetik, Bd. II, hg. v. R. Bubner, Stuttgart.

Le Brun, Jean (2002): Du pur amour. De Platon à Lacan, Paris: Seuil.

Leupin, Alexandre (1993): Fiction et incarnation. Littérature et théologie au Moyen Age, Paris: Flammarion. 
Luther, Martin (1972): Die gantze Heilige Schrifft Deudsch, hg. v. H. Volz, Herrsching.

\section{NOTES}

1. Brief an Louise Colet, 4.9.1852, Flaubert (1980), S. $151 \mathrm{f}$.

2. Dem entspricht die These Jean Le Bruns (2002), nach der der Diskurs des pur amour von der Theologie in die Literatur einwanderte. Er illustriert dies am Beispiel des päpstlichen Breves Cum alias, mit dem Innozenz XII. 1699 Fénelons zwei Jahre zuvor erschienene Explication des maximes des saints verwarf. Die Theologie des pur amour sei mit dieser Verwerfung keineswegs an sein Ende gekommen; die Vorstellung des pur amour sei in ein anderes Medium, den literarischen und den psychoanalytischen Diskurs nämlich, eingewandert. Flaubert beansprucht hier ganz selbstverständlich Ähnliches: Nicht mehr die Kirche, nicht mehr das theologische Schrifttum, sondern die moderne Dichtung - eben unser aller Körper - ist der Ort des Eingedenkens, des Nachvollzugs, des Bereitens des Liebesopfers.

3. Hegel (1971), S. 576.

4. Hegel (1971), S. 581.

5. Hegel (1971), S. 582.

6. Vgl. den Beitrag von Ph. Dufour in diesem Dossier („Salammbô, un tigre de marbre“), der sich der verstörenden Gewaltdarstellung in Salammbô widmet. Im gänzlichen Ausbleiben ironischer Erzählerkommentare sieht er eine Art Pathos zweiten Grades am Werk, welches eine ästhetische Affizierung durch den Gegenstand anzeigt.

7. Hegel (1971), S. 578.

8. Blumenberg (1964), S. 9-27.

9. Culler (1975), S. 38 f. und 193.

10. Auerbach (1958), S. 53.

11. Auerbach (1946), S. 433.

12. Leupin (1993), S. 10.

13. Zitiert nach Leupin (1993), S. 11.

14. Zur Darstellung der Figur Christi in Flauberts Tentation de saint Antoine und in den Trois contes im Kontext der religionsgeschichtlichen Ansätze Max Müllers und Ernest Renans siehe den Beitrag von Sylvie Triaire (»Le défilé des dieux, de l'idole primitive à Loulou«) in diesem Dossier. 15. Auerbach (1958), S. 42, zitiert Augustinus, Enarrationes in Psalmos 96.4 (CCSL 39, 1356-57; PL 37, 1239), den ich paraphrasiere.

16. In der Übersetzung Luthers: »Ejn jglicher sey gesinnet / wie Jhesus Christus auch war / Welcher / ob er wohl in göttlicher gestalt war / hielt ers nicht für einen Raub / Gotte gleich sein / Sondern eussert sich selbs (sed semet ipsum exinanivit) / vnd nam Knechts gestalt an / ward wie ein ander mensch / vnd an geberden als ein Mensch erfunden / Ernidriget sich selbs / vnd ward gehorsam bis zum Tode / ja zum tode am Creutz.« Nach Luther (1972), Bd. 2, S. 2367.

17. Vgl. B. Vinken: "Trois contes. Sermo humilis und Christentum« in diesem Dossier sowie Cécile Matthey, die das Mysterium der Inkarnation im Begriff der Gastfreundschaft fasst, wodurch die Idee der Verbreitung und des Teilens, kurz: die Idee des christlichen Universalismus, ins Zentrum rückt. 
INDEX

Mots-clés : réalisme, poésie moderne, christianisme, néant, Hegel, romantisme

Schlüsselwörter : Realismus, moderne Dichtung, Christentum, Nichtigkeit, Hegel, Romantik

\section{AUTEUR}

\section{BARBARA VINKEN}

Barbara Vinken ist Professorin für Allgemeine Literaturwissenschaft und Romanische Philologie an der Ludwig-Maximilians-Universität München (LMU). 